\title{
Training osteopathic medical students in behavioral medicine and psychiatry
}

JED MAGEN, DO

Medical students need more comprehensive training in behavioral sciences to cope with the increasing acuity of patients seen in an ambulatory-care setting. Present-day psychiatry teaching and clinical rotations emphasize severe psychopathologic disorders and inpatient care. Colleges of osteopathic medicine should integrate behavioral sciences into the preclinical curriculum and clinical training. Clinical psychiatry training should prepare students to care for patients with the kinds of behavioral difficulties seen in primary care settings in an integrated manner.

(Key words: Primary care, ambulatory care, psychiatry, behavioral sciences, behavioral medicine, biopsychosocial model, osteopathic medical education)

Medical educators face continuing challenges in preparing osteopathic medical students to practice in an ever-changing health care environment. Space limitations preclude examining the deficits of modern-day practitioners in the area of behavioral sciences and psychiatry. However, the medical literature suggests that physicians do not adequately recognize or treat patients with primary psychiatric disorders, psychiatric disorders as comorbid conditions, or other behavioral disorders. ${ }^{1,2} \mathrm{Al}$ -

Dr Magen is director of psychiatry residency training at Michigan State University-College of Osteopathic Medicine.

Reprint requests to Jed G. Magen, DO, Director of Residency Training, Michigan State University-College of Osteopathic Medicine, Department of Psychiatry, B-105 West Fee Hall, East Lansing, MI 48824. though it might be argued that osteopathic physicians talk to patients more and spend more time with them, this time spent may not equate with improved care or a greater devotion to psychosocial issues.

An examination of the problems presented by the traditional curriculum in educating students in the behavioral sciences is presented here, followed by suggestions for alternative approaches. Preclinical and clinical curricula are considered separately.

Throughout this paper, the terms psychiatry, behavioral science, and behavioral medicine will be used. As such, psychiatry will be defined as "the branch of medicine dealing with the study, treatment, and prevention of mental illness." 3

Behavioral medicine encompasses a large variety of techniques capable of altering behaviors to reduce known risk factors for illness. These techniques may also be used as preventive measures. For example, biofeedback can be used to reduce hypertension; other techniques may help to reduce or eliminate smoking. ${ }^{4}$ Behavioral sciences is the study of human and animal behavior applying experimental and observational methods similar to those found in other natural sciences (letter, T. Stachnik, PhD, December 1990).

\section{Preclinical medical education}

During preclinical medical education, students begin to develop attitudes and behaviors that will remain with them throughout their careers. They learn the primacy of the basic sciences and the premium placed on memorization. ${ }^{5,6}$ Through interviewing courses, students 
not previously oriented toward talking with patients and thinking about behavioral issues may become transiently interested in this area. However, as they become involved in the remainder of the curriculum, students can easily lose interest in behavioral issues. Such study areas occupy only a small portion of the compartmentalized curriculum. Silverman and colleagues ${ }^{7}$ present strong evidence for this phenomenon in their study involving students exposed to a course designed specifically to orient them toward psychiatric concepts and the interaction of biologic, psychologic, interpersonal, and sociocultural factors. Specific attention was given to developing an "integrated conception of the patient and his or her illness based on an understanding of important biologic, psychologic, interpersonal, and sociocultural factors."7 Little change occurred in students' strong biomedical orientation, despite their rating the course as extremely effective in conveying a unified concept of the patient. ${ }^{7}$

More recently, this unified model has been described as the "biopsychosocial model."8 Most teaching in the first 2 years emphasizes the "bio" segment of this model almost exclusively. Basic science faculty often have little knowledge of, or incentive to discuss, behavioral issues. Many clinical faculty are not oriented toward including discussions of psychologic and social factors in illness in their lectures. The other usual venue for behavioral science teaching is a series of lectures on psychopathology. This format emphasizes memorization. However, it fails to convey the richness of human behavior and makes no connections with clinical skills needed to uncover behavioral disorders. Furthermore, psychiatry courses often do not relate to other courses in any logical way, as would a pulmonary course to a cardiology course, for example.

\section{The clinical curriculum}

Most colleges of osteopathic medicine (COMs) operate on a clinical teaching model similar to that of allopathic institutions. Few COMs have their own academic teaching institutions; rather, autonomous, geographically separate community hospitals are used for rotations. This model has both strengths and weak- nesses. On the positive side, students are generally exposed to less subspecialized care. More of the clinical pathology relates to what is commonly seen in the community. However, a disconnection may occur between what the COM administrators think is happening educationally to their students and what really occurs.

In the community hospitals, attending physicians are not usually college faculty members, and they may not be available because of the demands of private practice. Similarly, house staff may not be available to provide further teaching.

Psychiatry is often taught in a 4- to 6-week block on an inpatient psychiatry unit. These rotations are unlikely to provide the kind of training useful to primary care physicians. Students encounter those patients with the most severe psychopathologic disorders, such as schizophrenia, bipolar disorders, and severe major depression. No opportunity exists for students to see patients with mild-to-moderatebehavior disorders. It is unusual for students to gain outpatient experience.

On many rotations, students seem to be silent partners, passively following the attending physician. Yager and coauthors ${ }^{9}$ studied students' attitudes at three medical schools. Contact with attending psychiatrists, close supervision of their clinical work, and close contact with psychiatric residents regarding psychiatry clerkships, seemed most important to students. Negative evaluations were common when the primary experience was with chronic, longer-term inpatients, when the psychiatrist and other mental healthcare professionals did not have well-delineated roles, and when the attending psychiatrist was mainly in an administrative role. ${ }^{9}$

Many of our osteopathic psychiatry rotations may not be meeting students' perceived needs. Because most students will not become psychiatrists, they need programs that meet reasonable educational objectives.

\section{Curriculum changes}

Curriculum changes might first occur in the following areas:

- First and most fundamentally, for what role are we educating students? 
Table

Swampscott Study ${ }^{10}$ : Objectives for Medical Education in the Behavioral Sciences

\section{Objectives}

Increase student, faculty, medical community's awareness of health, disease; enrich physician/patient empathy.

Interdependence of biologic, psychologic, social, environmental, phenomena in determining:

- Pathogenesis, distribution, diagnosis, management of specific disease

- Potentialities for disease prevention/modification

- Beliefs, attitudes, values associated with human response to general, specific illness

Historic, cultural/comparative perspectives: Basis for considering impact of technologic, social change on:

- Contemporary, future society

- Contemporary, future medical science, forms of medical care

Communication processes, basic human relationships between healthcare personnel patients and healthcare personnel among themselves; incorporating communication skills in medical-setting activities

Universities, hospitals, other healthcare organizations' social structure: Factors governing health resources organization, distribution to the community

\section{Knowledge needed to achieve objectives}

Neural-Behavioral Sciences

- Physiologic psychology (Neurophysiology)

- Chemical psychology (Neurochemistry)

- Behavioral pharmacology (Psychopharmacology)

- Behavioral genetics

- Embryologic, morphologic CNS development (Normal development)

Individual psychology

- Personality, personality development

- Concepts of affect, memory, learning, motivational drives, communication, consciousness, language in context of normal development

\section{Social/Cultural Sciences}

- Anthropology

- Sociology

- Ethology

- History (Organization of humans)

- Economics

- Political science

- Demography

disorders. As such, physicians will have to make treatment decisions or refer patients based on, perhaps, a fragmentary understanding of what is troubling their patients.

Likewise, many behavioral problems occur in an ill-defined manner, not amenable to categories defined in the Diagnostic and Statistical Manual of Mental Disorders, third edition, revised (DSM-III-R). Students need to recognize common behavioral problems and to treat them. They will need to recognize, or be suspicious of, less common and more severe problems and make appropriate referrals. More fundamentally, students need a basis for thinking about behavior in an organized fashion. In this way, students can put symptoms together in a meaningful way for a diagnosis and develop a treatment plan. Given our present methods of training physicians, it is difficult to argue that we are, in fact, preparing them to practice medicine in any systematic or effective way.

Objectives for behavioral science education have been available for years. In many respects, the skills and the general categories thought important have remained similar throughout the years. The 1966 Swampscott study ${ }^{10}$ outlined objectives and a body of knowledge for behavioral science edu-

- What do we want students to learn? What are their learning objectives?

- What are the most effective means of teaching, given the available resources?

Nonpsychiatric physicians will see patients with ill-defined behavioral complaints, poor coping skills, and various reactions to physical cation during medical school that still seems current and relevant (Table). Overly inclusive, this list may be beyond the resources of many osteopathic medical institutions to include didactic education in all these areas. However, the list serves as a useful starting point in curriclum design for behavioral science education. 


\section{Preclinical changes}

Given the objectives in the Table, some courses must be offered with specific behavioral sciences content. Interviewing skills, ethics of relationships, and an understanding of the physician-patient relationship should be taught in the first year. Preferably, patient contact will be included in such courses. Many schools teach this course in a small-group format. ${ }^{7,11}$ In the second year, a course in behavioral medicine and psychiatry reinforces a holistic approach to patient care and conveys much of the material found in the Table.

To understand human behavior, one has to understand developmental processes, both psychologic and biologic. I propose that a course in development be used as the basic template for an integrated approach to human behavior. For example, adolescent development can be approached from neurochemical-neurophysiologic perspectives (hormonal regulation, the increased activity of the hypothalamic-pituitary-adrenal-gonadal axes); from psychologic perspectives (separation-individuation, family responses to separation); from social perspectives (societal response to adolescence, school issues); from a psychopathologic perspective (substance abuse, depression, and suicide); and from a health maintenance perspective (cigarette smoking, dieting, and so on). This approach proceeds logically from intrauterine development to senescence, and it continues to emphasize integration at several levels, as well as holistic care. Faculty members from a number of different departments could contribute. Pediatricians, neurologists, neuroanatomists, psychologists, and social workers may be involved. Such a course may serve as the critical centerpiece of the preclinical behavioral science curriculum because it attempts to integrate basic science material, developmental approaches, and clinical material. In preparing students for clinical training, this course should foster an appreciation for the complex biopsychosocial nature of behavior and illness. Other areas featured in the Table can be included as separate topics, as time and resources permit.

Beyond specific courses, behavioral science teaching should reflect the reality of medical practice. Psychosocial issues must be introduced as a routine part of clinical courses in all specialties because they are present in practice. However, it is not enough simply to have a psychiatrist give one or two lectures. Frequent reinforcement and continued discussion of behavioral factors in disease must continue throughout the entire curriculum. Gorlin and Zucker ${ }^{12}$ describe such an approach used throughout the 4-year curriculum at the Mount Sinai School of Medicine in New York, NY.

Role models play a powerful force in developing students' attitudes and in influencing their specialty choice. Just as residents selectively model desired traits from different faculty members, so the process is likely to remain similar among medical students. Faculty members who present a truly integrated approach to illness and who are aware of psychosocial factors in disease are ideal for role models. The more faculty members with this orientation, the greater the probability that students will model their attitudes and behaviors after such individuals.

\section{Clinical training changes}

Clinical training should flow from the aforementioned base. The teaching of behavioral disorders should be included on all rotations. Consider the following example:

A 74-year-old white woman was being treated in the hospital for coronary artery disease. Angina, usually on exertion but at times at rest, and exertional dyspnea were her major symptoms. She also had fairly well-controlled hypertension. Students, interns, residents, and an attending physician on a general medicine rotation saw the woman. She often complained to her medical team of pain during the previous night. She frequently voiced fears of dying and was apprehensive about going home. She often complained of being lonely. Without much comment from anyone concerning these behavioral issues, the patient was discharged to home.

In later conversations with the medical students involved in this case, it was learned that the patient lived alone, had no family, and that neighbors intermittently cared for her. 
When asked to speculate about diagnostic entities that might fit these observations and information, the students readily volunteered that this patient was probably anxious about her symptoms and about going home. She may have been anxious about being alone in the hospital and may have complained of her symptoms to prolong any visits by healthcare personnel. The students were concerned that this patient had little support at home and wondered if she would comply with her medication regimen.

The group discussed techniques of eliciting information related to these areas of concern. With help, the students generated a list of other interventions, including visiting nurses, frequent outpatient appointments, a possible move to a supervised care situation, and a suggested referral to a senior care center. The role of the physician and of other personnel, including hospital social workers, was discussed.

Students were able to make a series of behavioral diagnoses retrospectively, and develop a plan to address these concerns. They suggested potentially quite effective, and not very complex, psychosocial interventions that may be extremely valuable to this patient. These interventions can be accomplished during the hospitalization if someone takes the time to think about the behavioral issues involved. If consistently presented (and this is a critical variable), students may readily pick up this orientation.

Most psychiatry rotations should be designed with the nonpsychiatric physician in mind. The kind of case previously described is far more common than ones involving the acute schizophrenic patient in the medicalsurgical unit. Rotations in which students will have contact with patients with common disorders, such as outlined previously, might include outpatient psychiatry settings and consultation-liaison settings. Placing students in primary care settings with clinicians who are particularly skilled in psychosocial care is an underused option. In this setting, meetings for didactics and case discussions can take place with a clinically oriented psychologist or psychiatrist.

\section{Resource issues}

Few COMs have enough psychiatric faculty for preclinical teaching. Teaching on clinical rotations may occur hundreds of miles from the COM, in settings with few behavioral science resources. However, before labeling the whole enterprise as impractical, recognize that creative alternatives may exist. Primary care physicians who are psychosocially oriented can teach preclinical topics, including interviewing skills. Pediatricians and psychologists can teach courses in human development. Similarly, nurses, sociologists, social workers, and persons from other disciplines could teach certain aspects of these courses. Some faculty members will always be impervious to entreaties from above to be psychosocially aware. Thus, faculty development - otherwise known as education-remains an important component in developing such a curriculum.

Because of the geographic disconnection between clinical training sites and COMs, changes in clinical training seem more difficult. At a minimum, the COMs should be able to bring in a mental health clinician, preferably a psychiatrist, to hold clinically oriented seminars with students. Conducting rounds with students in the outpatient or inpatient setting would be better.

Gifted physicians who know how to speak to patients' psychosocial issues, as well as organic issues, do exist in the communities. With some backup from psychiatrists in a didactic and advisory capacity, such individuals make these rotations more useful than rotations in inpatient psychiatry units. For many students, such rotations could replace psychiatry rotations.

\section{Organizational issues}

With this kind of integrated program, a single administrative unit must have control of the entire 4-year behavioral science training program. Psychiatry departments are the logical units in which to place such programs. Schools without a psychiatry department may want to give administrative control to family practice departments. As with any enhanced teaching or administrative load, compensation and rewards (or both) are desirable means to acknowledge faculty efforts. ${ }^{9}$ 
Obviously, many variations exist on the themes presented herein. Curricula will vary, depending on the unique combinations of faculty and clinical resources, interest, and other factors at the different colleges.

\section{Comment}

Osteopathic medicine is, and has been, largely a primary care profession. It has always emphasized a comprehensive approach to patient care. Truly relevant, helpful behavioral science training for our students, then, can only strengthen what we as a profession already do best.

\section{References}

1. Daniels RS: Is psychiatry a "primary" medical care specialty? Psychiatric Opinion 1976;13:34-36.

2. Chang G, Warner V, Weissman MW: Physicians' recognition of psychiatric disorders in children and adolescents. Am J Dis Child 1988;142:736-739.

3. Dorland's Illustrated Medical Dictionary, ed 25. Philadelphia,
Pa, WB Saunders, 1974, p 1281.

4. Birk L: Behavior therapy and behavioral psychotherapy, in Nicholi AM, Jr (ed): The New Harvard Guide to Psychiatry. Cambridge, Mass, Belknap Press, 1988, pp 534-562.

5. Clawson DK: The education of the physician. Academic Medicine 1990;65:84-88.

6. Lewis IJ, Sheps CG: The Sick Citadel: The American Academic Medical Center \& the Public Interest. Cambridge, Mass, Oelgeschlager, Gunn \& Hain, Inc, 1983.

7. Silverman D, Gartrell N, Aronson M, et al: In search of the biopsychosocial perspective: An experiment with beginning medical students. Am J Psychiatry 1983;140:1154-1159.

8. Engel GL: The clinical application of the biopsychosocial model. Am J Psychiatry 1980;137:535-544.

9. Yager J, Lamotte K, Nielsen A III, et al: Medical students' evaluation of psychiatry: A cross-country comparison. Am J Psychiatry 1982;139:1003-1009.

10. Beck JC: A study of behavioral sciences in medicine, in Cope $\mathrm{O}$ (ed): Man, Mind \& Medicine: The Doctor's Education. Philadelphia, Pa, JB Lippincott Co, 1968, pp 98-104.

11. Taylor KM, Shapiro M, Kelner M: The integration of behavioral science theory and clinical experience for second-year medical students. J Med Educ 1983;58:353-355.

12. Gorlin R, Zucker HD: Physicians' reactions to patients: A key to teaching humanistic medicine. $N$ Engl $J$ Med 1983;308:1059-1063.

13. Bucher R, Stelling JG:Becoming Professional. Beverly Hills, Calif, Sage Publications, 1977. 